\title{
Dark Energy Faces Multiple Probes
}

\begin{abstract}
The Dark Energy Survey has combined its analysis of four cosmological observables to constrain the properties of dark energy-paving the way for cosmological surveys that will run in the next decade.
\end{abstract}

\section{by Nikhil Padmanabhan*}

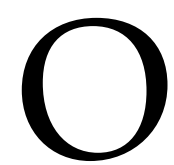
ne of the top goals in cosmology today is understanding the dark energy that is responsible for the accelerated expansion of the Universe. Is the dark energy consistent with the cosmological constant of general relativity-representing a constant energy density filling space homogenously? Or can we find deviations from general relativity on cosmological scales that suggest a more complex nature for gravity? Questions like these motivate the current and next generations of surveys that aim to map out ever larger volumes of the Universe, using a wide variety of probes to constrain the properties of dark energy. The Dark Energy Survey (DES) has now derived such constraints from the combined analysis of four canonical observables related to dark energy: supernovae, baryon acoustic oscillations, gravitational lensing, and galaxy clustering [1]. The resulting bounds confirm

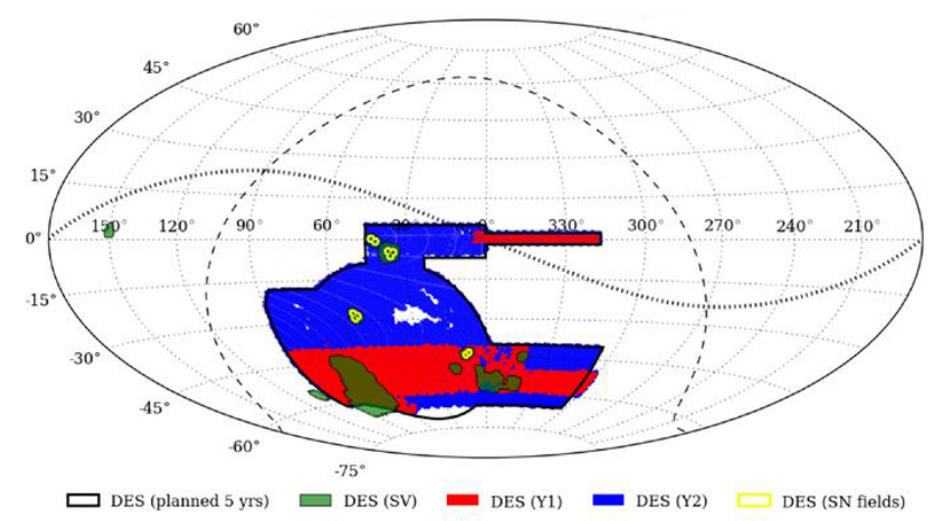

Figure 1: Area of the sky observed by the Dark Energy Survey in its 5-year mission. The different colors indicate different observation runs. SV: preliminary science verification run; $Y 1$ : year 1; Y2: year 2; $\mathrm{SN}$ fields: areas containing the supernovae used as standard candles to monitor the Universe's expansion. ( $F$. B. Abdalla et al., Mon. Not. R. Astron. Soc. 460, 1270 (2016)) *Department of Physics and Department of Astronomy, Yale Uni-
versity, New Haven, CT, USA what we knew from previous studies, which focused on single probes. But the results indicate that this multiprobe approach could allow surveys in the 2020s to improve such constraints by orders of magnitude, possibly bringing us close to solving the dark energy puzzle.

Measurements of dark energy traditionally come in two "flavors." The first measures the geometric expansion of the Universe. It includes observations of supernovae and of baryon acoustic oscillations (BAO). Type Ia supernovae can be used as "standard candles" - their known brightness allows astronomers to estimate their distance. In the 1990s, measurements of the recession speed (or redshift) of supernovae as a function of distance led to the discovery that the expansion of the Universe was accelerating, upsetting predictions based on models of a matter-dominated Universe [2]. BAO are also related to spatial distances, but they serve instead as "standard rulers" that can calibrate cosmic lengths. BAO are fluctuations in the density of matter caused by acoustic waves in the primordial photon-baryon plasma of the early Universe. The length of the BAO standard rulers can be estimated from cosmic microwave background $(\mathrm{CMB})$ measurements. By observing the angular size of the $\mathrm{BAO}$ rulers at different times, scientists can directly measure the geometric background expansion of the Universe.

The second flavor of measurements focuses on dark energy's effect on the growth rate of cosmic structures, both visible and dark. The accelerated expansion causes these structures to grow slower, as gravity has less time to pull in matter around over-dense regions. The growth suppression can be characterized by observing the distribution of matter. The DES traces the matter distribution through weak lensing-measurements of subtle, coherent distortions of the shapes of background galaxies due to the gravitational lensing of foreground matter. From such distortions, the distribution of foreground matter (which is mostly dark matter) can be inferred. The small magnitude of the weak-lensing effect makes it challenging to measure and susceptible to systematic observational errors, but a number of surveys, including the DES [3], were able to produce maps of the matter distribution using weak lensing.

Another approach to measure structure-growth suppression is to trace out the matter distribution by mapping out the positions of visible galaxies. Measuring distances to galaxies requires determining their redshifts through spec- 
troscopy. While the DES doesn't have high-resolution spectroscopy capabilities, it partially addresses this challenge by imaging the sky through five spectral filters, acquiring lowresolution spectra that provide approximate measurements of distances to galaxies. These distance errors blur out the three-dimensional structure, but the resulting maps are still a powerful probe of gravitational structure formation. While these galaxy maps are less noisy than the weak-lensing maps, they are limited because we don't know exactly how dark matter is distributed with respect to visible galaxies. However, the DES Collaboration has developed techniques based on correlating weak-lensing data with visible galaxy distributions, which allows it to use the higher signal-tonoise galaxy maps for cosmology.

Imaging a large area of the Southern Sky (Fig. 1) with the 4-m Victor M. Blanco Telescope at Cerro Tololo InterAmerican Observatory in Chile, the DES Collaboration has, for the first time, combined all four dark energy probes in a single analysis. The primary results of this work are independent constraints on the cosmological densities of both dark matter and dark energy (Fig 2). The DES Collaboration also constrains the dark energy equation of state parameter $(w)$. As Fig. 2 shows, these constraints are not yet competitive with the best constraints derived from other experiments that combine galaxy surveys with $\mathrm{CMB}$ data. However, demonstrating the feasibility of a multiprobe approach within a single survey is, in my view, the most important aspect of the work, as it has several advantages over single-probe surveys. First, since all the probes derive from a single survey, calibrations and systematic errors can be consistently controlled across the multiple probes-a harder task for studies that compile data from separate experiments. Second, the Collaboration can uniformly apply the same blinding strategy-an analysis approach in which information is withheld from the researchers carrying out the analysis to reduce observer bias. Finally, the simultaneous characterization of the four probes allows the survey to carry out the cross-correlation analyses described above. While all of the above is, in principle, possible using separate experiments, in practice it is extremely challenging. What's more, the richness of the data from a multiprobe analysis may inspire novel tests of gravity and dark energy that hadn't been previously thought about.

The results of the DES multiprobe analysis bode very well for the next decade of dark energy research. In the short term, we can expect the DES Collaboration to significantly improve on its constraints. The data analyzed in this work represent only a fraction of the overall data that the DES has already taken. The final dataset will have three times more data for the weak-lensing and galaxy-clustering measurements and include 10 times more supernovae. Statistically, this should yield a factor of 2-4 improvement in the constraints presented here. As constraints get tighter, tensions among different observables could potentially signal cracks

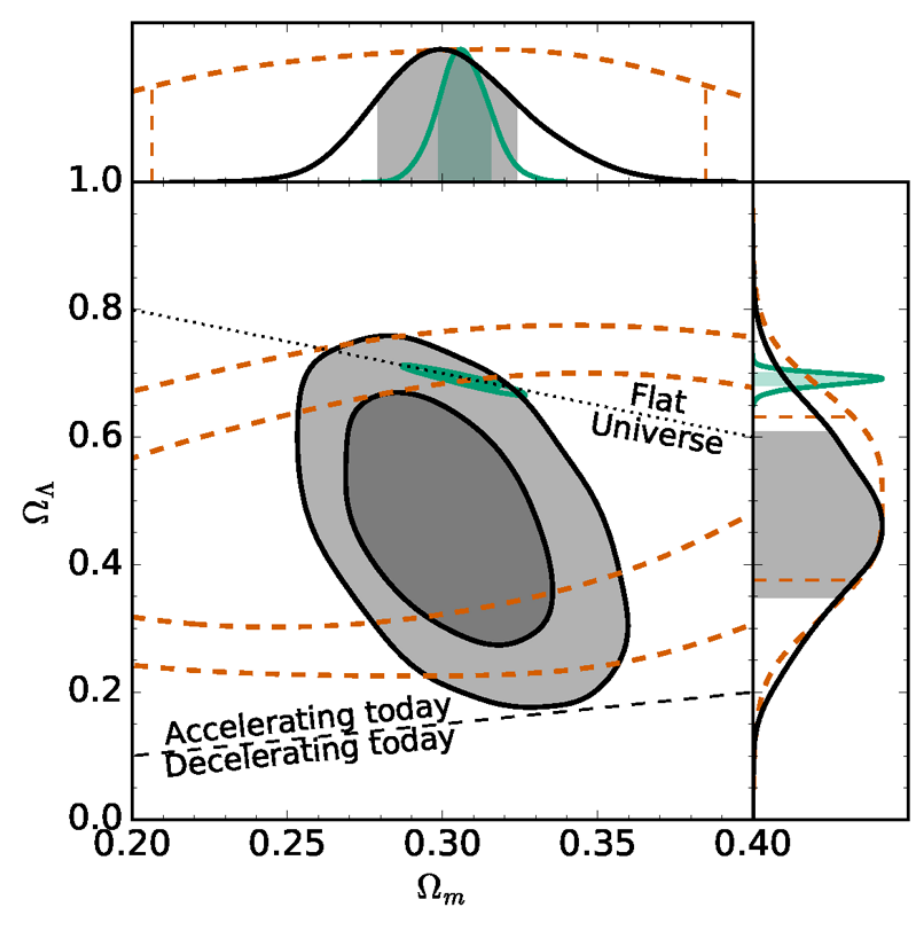

Figure 2: Constraints on dark energy density $\left(\Omega_{\Lambda}\right)$ and on matter density $\left(\Omega_{m}\right)$. Gray contours are constraints from DES data on weak gravitational lensing, large-scale structure, supernovae, and $\mathrm{BAO}$. Green contours are the best available constraints, derived from $\mathrm{CMB}$, supernovae, and BAO data. Contours represent $68 \%$ and $95 \%$ statistical confidence. (Y. Guo et al., Phys. Rev. Lett. (2019))

in existing cosmological models. There are already intriguing disagreements between local measurements of the Hubble constant compared with its value inferred by $\mathrm{CMB}$ and $\mathrm{BAO}$ [4], as well as hints of disagreements on the amount of structure revealed by $\mathrm{CMB}$ and weak lensing [5]. The full DES analysis may help resolve these discrepancies, or possibly-and that would be even more exciting-exacerbate them.

In the longer term, we can look forward to results from a number of similarly powerful ongoing surveys, and from even larger surveys planned over the next decade. These include photometric surveys like the DES (such as the KiloDegree Survey, the Hyper Supreme-Cam Survey, and the Large Synoptic Survey Telescope), spectroscopic surveys (the Extended Baryon Oscillation Spectroscopic Survey and surveys planned with the 4-m Multi-Object Spectroscopic Telescope and the Dark Energy Spectroscopic Instrument), two satellite missions that will combine both photometric and spectroscopic observations (the Euclid telescope and the Wide Field Infrared Survey Telescope), CMB measurements (the Simons Observatory and the Stage- 4 CMB experiment), and surveys that use newer probes like the $21-\mathrm{cm}$ hydrogen 
line and gravitational waves. The breadth of these programs ensures that the DES measurements are just the beginning of an exciting exploration of one of the most intriguing cosmological questions.

This research is published in Physical Review Letters.

\section{REFERENCES}

[1] T. M. C. Abbott et al., "Cosmological constraints from multiple probes in the Dark Energy Survey," Phys. Rev. Lett. 122, 171301 (2019).

[2] A. G. Riess et al., "Observational evidence from supernovae for an accelerating universe and a cosmological constant," Astron. J. 116, 1009 (1998); S. Perlmutter et al., "Discovery of a supernova explosion at half the age of the Universe," Nature 391, 51 (1998); S. Perlmutter et al., "Erratum: Discovery of a supernova explosion at half the age of the Universe," Nature 392, 311 (1998).
[3] M. A. Troxel et al., "Dark Energy Survey Year 1 results: Cosmological constraints from cosmic shear," Phys. Rev. D 98 (2018).

[4] Wendy L. Freedman, "Cosmology at at crossroads: Tension with the Hubble Constant," arXiv:1706.02739.

[5] H. Hildebrandt et al., "KiDS-450: cosmological parameter constraints from tomographic weak gravitational lensing," Mon. Not. R. Astron. Soc. 465, 1454 (2016); E. van Uitert et al., "KiDS+GAMA: cosmology constraints from a joint analysis of cosmic shear, galaxy-galaxy lensing, and angular clustering," Mon. Not. R. Astron. Soc. 476, 4662 (2018); S. Joudaki et al., "KiDS-450 + 2dFLenS: Cosmological parameter constraints from weak gravitational lensing tomography and overlapping redshift-space galaxy clustering," Mon. Not. R. Astron. Soc. 474, 4894 (2017); C. Hikage et al., "Cosmology from cosmic shear power spectra with Subaru Hyper Suprime-Cam first-year data," Publ. Astron. Soc. Jpn. 71, 43 (2019); C. Chang et al., "A unified analysis of four cosmic shear surveys," Mon. Not. R. Astron. Soc. 482, 3696 (2018).

10.1103/Physics. 12.48 